\title{
Comparison of minimally invasive versus open transforaminal lumbar interbody fusion in two-level degenerative lumbar disease
}

\author{
Guangfei Gu • Hailong Zhang • Guoxin Fan • \\ Shisheng He $\cdot$ Xiaobing Cai $\cdot$ Xiaolong Shen • \\ Xiaofei Guan • Xu Zhou
}

Received: 22 October 2013 / Accepted: 23 October 2013 / Published online: 17 November 2013

(C) Springer-Verlag Berlin Heidelberg 2013

\begin{abstract}
Purpose The purpose of this study was to compare the clinical and radiological outcomes of minimally invasive versus open transforaminal lumbar interbody fusion in two-level degenerative lumbar disease.

Methods We conducted a prospective cohort study of 82 patients, who underwent two-level minimally invasive or open transforaminal lumbar interbody fusion (TLIF) from March 2010 to December 2011. Forty-four patients underwent minimally invasive transforaminal lumbar interbody fusion (MITLIF) (group A) and 38 patients underwent the traditional open TLIF (group B). Demographic data and clinical characteristics were comparable between the two groups before surgery $(p>0.05)$. Peri-operative data, clinical and radiological outcomes between the two groups were compared.

Results The mean follow-up period was $20.6 \pm 4.5$ months for group A and 20.0 \pm 3.3 months for group B ( $>>0.05)$. No significant difference existed in operating time between the two group $(p>0.05)$. X-ray exposure time was significantly longer for MITLIF compared to open cases. Intra-operative blood loss and duration of postoperatively hospital stay of group A were significantly superior to those of group B ( $p<$ 0.05 ). On postoperative day three, MITLIF patients had significantly less pain compared to patients with the open procedure. No statistical difference existed in pre-operative and latest VAS value of back pain (VAS-BP) and leg pain (VAS-LP),
\end{abstract}

Guangfei Gu and Hailong Zhang contributed equally to this work.

G. Gu $\cdot$ H. Zhang $\cdot$ G. Fan $\cdot$ S. He $(\bowtie) \cdot$ X. Cai $\cdot$ X. Shen •

X. Guan $\cdot$ X. Zhou

Department of Orthopaedics, Shanghai Tenth People's Hospital,

Tongji University School of Medicine, Yanchang Road 301,

Shanghai 200072, People's Republic of China

e-mail: tjsyorthop@163.com pre-operative and latest ODI between the two groups. The fusion rate of the two groups was similar $(p<0.05)$. Complications included small dural tear, superficial wound infection and overlong screws. When comparing the total complications, no significant difference existed between the groups $(\mathrm{p}>0.05)$. Conclusions MITLIF offers several potential advantages including postoperative back pain and leg pain, intra-operative blood loss, transfusion and duration of hospital stay postoperatively in treating two-level lumbar degenerative disease. However, it required much more radiation exposure.

Keywords Transforaminal lumbar interbody fusion .

Minimally invasive $\cdot$ Two-level $\cdot$ Lumbar degenerative disease

\section{Introduction}

Lumbar fusion is a common surgical procedure for the treatment of symptomatic spinal pathologies, such as degenerative spinal disease, trauma, spondylolisthesis, and deformity $[1,2]$. Among various spinal fusion techniques, open transforaminal lumbar interbody fusion (TLIF) has been proven to be a safe technique to achieve lumbar fusion via a unilateral approach with minimal retraction of neural elements $[3,4]$. Nevertheless, like other open posterior procedures, one of the drawbacks of open TLIF is the iatrogenic lumbar soft tissue injury, which is supposed to relate to postoperative pain, long recovery time and impaired spinal function.

Minimally invasive transforaminal lumbar interbody fusion (MITLIF) has become a popular and well-established technique since its first description by Foley et al. in 2002 [5]. Compared with the open procedure, MITLIF appears to achieve similar fusion rates while minimizing blood loss and tissue injury, causing smaller wounds, increasing the speed of 
recovery, and reducing postoperative pain [6-9]. Currently, there are a few studies [7, 9-11] which have reported the outcomes and benefits of MITLIF. However, these studies mainly focus on one-level TLIF, and comparisons of minimally invasive versus open TLIF in two-level degenerative lumbar disease are very limited. To the best of our knowledge, this study may be the first study to compare the minimally invasive versus open TLIF in two-level degenerative lumbar disease.

\section{Materials and Methods}

\section{Clinical data}

This study was approved by the Committee of Medical Ethics and the institutional review boards of the authors' institutions. From March 2010 to December 2011, a total of 82 consecutive patients underwent two-level TLIF by one surgeon in our hospital. Patients admitted on odd-numbered days were assigned to the minimally invasive group, and those admitted on even-numbered days were assigned to the open group. Of all the patients, 44 patients underwent MITLIT (group A) and 38 patients underwent the traditional open TLIF (group B).

Demographics and procedure data are listed in Table 1. Indications for surgery were: (1) far-lateral disk herniation, huge lumbar disk herniation or recurrent lumbar disk herniation and symptomatic adjacent segment pathology/ degeneration; two-level lumbar stenosis both requiring facetectomy and fusion; symptomatic lumbar stenosis with degenerative disc disease combined with segmental instability; symptomatic degenerative disc disease; (2) two-level fusions were needed between L3 and S1; (3) persistent or recurrent low back pain or leg pain lasting at least six months and resulting in a significant reduction of quality of life, despite conservative therapy, including physical therapy and pain management.

Contraindications were: (1) patients associated with previous spinal surgery, lumbar fracture, active infection, severe osteoporosis and severe obesity; (2) combination of coronal and/or sagittal deformities that needed a surgical correction; (3) degenerative spondylolisthesis with major instability or isthmic spondylolisthesis; (4) any major psychological problem. The inclusion criterion of segmental instability was more than $4 \mathrm{~mm}$ of translation or 10 of angular motion on preoperative flexion-extension radiographs.

\section{Surgical techniques}

\section{Minimally Invasive TLIF}

Under general anaesthesia, the patient was evenly placed in a prone position on a radiolucent operating table. The posterior superior iliac spines (PSIS) were palpated, and the lines connecting to the PSIS were labeled with a marker. Our selfmade pre-operative locator [12] was then placed on the patient's back with the central part in the midline over the approximate spinal levels of interest and fixed with an adhesive plaster. The anteroposterior (AP) fluoroscopic image was obtained. The targeted levels were confirmed according to the different markers on the locator. Based on the spatial relationship, the intervertebral spaces and the pedicle positions were marked on the body surface. An incision was planned by connecting a line between the outer portions of both ends of the pedicles (approximately $3.0 \mathrm{~cm}$ off midline) (Fig. 1). Then a skin incision about $3.0-4.0 \mathrm{~cm}$ was made on the more symptomatic side or more severe pathology side according to the images. The paravertebral muscles were split and retracted laterally to the outer edge of the facet joint, and the lamina and facet joint were exposed. Adequate decompression was achieved by cutting the inferior portion of the lamina, hypertrophied superior and inferior articular processes and the ligamenta flava. After complete decompression, the operating table or the expandable tubular retractor was tilted about $15^{\circ}$ to observe the contralateral side. Adequate bony decompression was carefully performed with a rongeur leaving the ligamentum flavum in place to protect the dural sac and the nerve root. Then the ligamentum flavum was removed en bloc with a curette. With recognition of the inner aspect of the
Table 1 Demographic data and clinical characteristics

\begin{tabular}{llll}
\hline Demographic data & Group A (n=44) & Group B (n=38) & P \\
\hline Mean age (years) & $66.4 \pm 6.7$ & $64.1 \pm 7.8$ & 0.16 \\
Duration of symptoms (months) & $45.1 \pm 24.2$ & $50.8 \pm 23.1$ & 0.29 \\
Gender (M/F) & $19 / 25$ & $15 / 23$ & 0.73 \\
Preoperative diagnosis & & & \\
Symptomatic degenerative disc disease & $15(34.1 \%)$ & $11(28.9 \%)$ & 0.62 \\
Symptomatic two-level lumbar stenosis & $18(40.9 \%)$ & $14(36.9 \%)$ & 0.71 \\
Symptomatic lumbar stenosis with segmental instability & $11(25.0 \%)$ & $13(34.2 \%)$ & 0.36 \\
Levels of fusion & & & \\
L3- L5/L4- S1 & $13 / 31$ & $14 / 24$ & 0.48 \\
\hline
\end{tabular}


Fig. 11 Palpate the iliac crests pre-operatively. 2 Make a mark on the overlying skin at the uppermost margin of each iliac crest. 3 Place the pre-operative grid locator on the patient's back with the centre in the midline over the approximate spinal levels of interest and fix with an adhesive plaster. 4 Obtain the AP fluoroscopic X-ray image involved in the sacrum. 5 Confirm the levels for surgery according to the different markers on the locator and mark the incision and pedicles

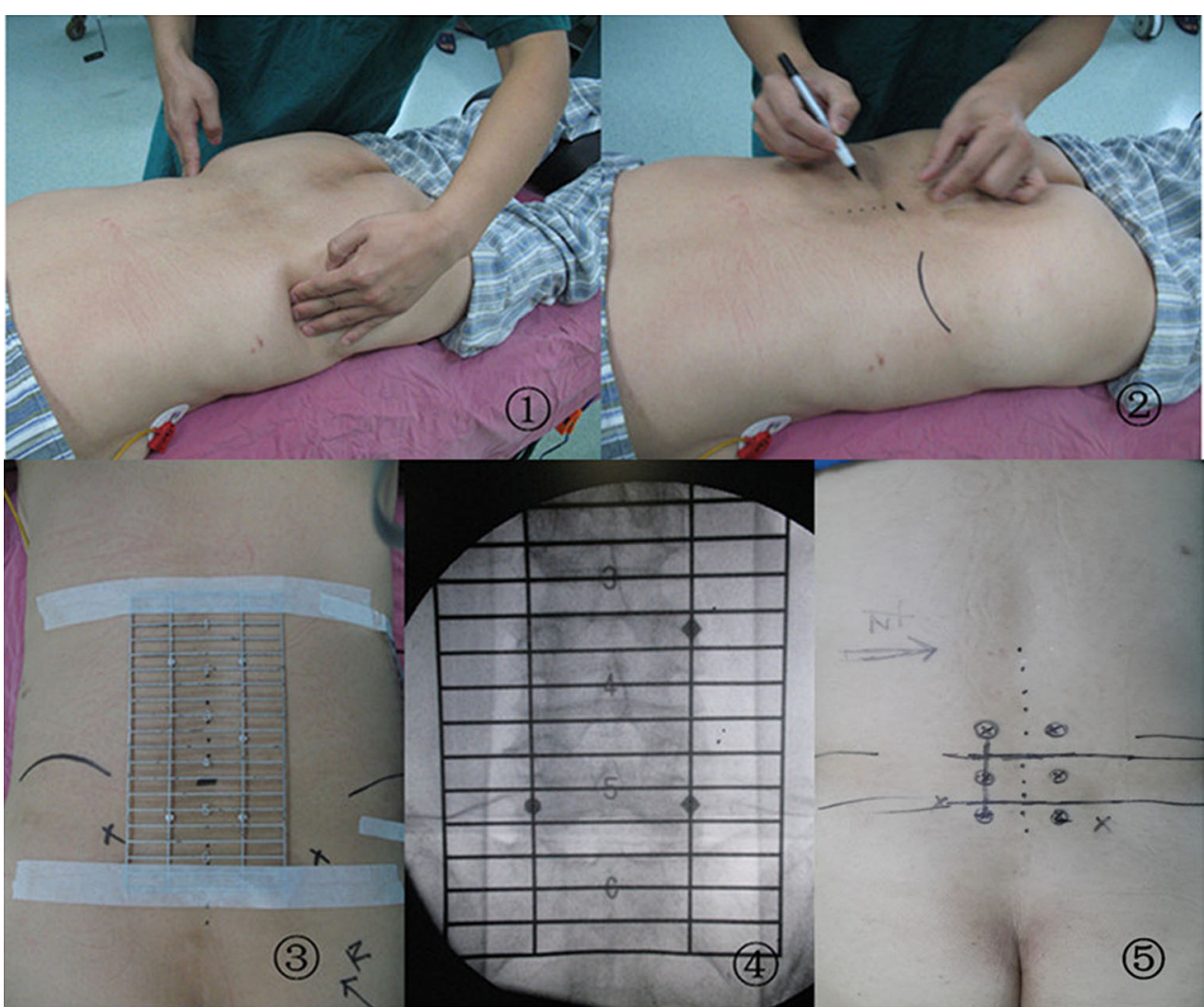

pedicle on the contralateral side, we confirmed adequate decompression of the contralateral side. Then the vertebral endplates were carefully prepared. Sufficient autologous bone graft obtained from the resected lamina and facet was packed in the anterior disc space. A single PEEK cage (Capstone Medtronic Sofamor Danek, Memphis, TN, USA) filled with autologous bone was inserted obliquely across the disc space. No additional contralateral facet fusion was performed in all the patients. The decompression and fusion procedure of another segment was the same as above mentioned.

Following the decompression and fusion procedure, the ipsilateral pedicle screws were placed in direct visual as in the open procedure, and the contralateral pedicle screws were percutaneously placed. Depending on the depth of the tissue between the skin and pedicle, a 1-2 cm lateral skin incision was made to insert the Jamshidi needle at an appropriate angle into the pedicle. Using the Jamshidi needle, the AP and lateral images were obtained to localize the needle tip, docked against the bone at the junction of the base of the transverse process and facet joint. The needle was then gently tapped with a mallet to engage the tip in the bone. Fluoroscopy was intermittently used to confirm the direction and the depth, to make sure the needle remained lateral to the medial pedicle wall. As the needle advanced to cross the pedicle center, a guidewire was inserted through the cannula into the pedicle. The cannula was then carefully removed, leaving the guidewire in place. The fascia and muscle were dilated to allow screw placement. After preparation of the pedicle with a tap using cannulated taps, the percutaneous cannulated pedicle screw-rod system (Sextant; Medtronic Sofamor Danek, Memphis, TN, USA) was placed.

\section{Open TLIF}

The open TLIF approach was performed using a midline open approach. A more detailed description of the procedure is available in the literature $[7,13,14]$.

\section{Clinical and radiological evaluation}

The data collected for analysis were operating time, intraoperative blood loss, total amounts of transfusion, X-ray exposure time, duration of hospital stay, clinical and radiographic results and complications. The patients were asked for follow-up at three, six and 12 months postoperatively and annually thereafter. Back and leg pain were quantified by visual analog scores (VAS) collected from the patients preoperatively, three days postoperatively and at last follow-up. The Oswestry Disability Index (ODI), version 2.0, was used before and after surgery to provide information about how the patient's leg (or back) condition has affected his/her management in daily life.

Radiographs (AP and lateral images) were taken to evaluate the fusion status at 12 months postoperatively. When it is 
difficult to confirm the fusion status by radiographs, CT scan with two-dimensional reconstruction was performed. The radiographic assessment of solid fusion was performed by two independent, experienced spine surgeons who were not involved with the surgical procedures. The fusion status was assessed using plain radiographs, according to the Bridwell's posterior fusion grades [15], namely, grade I: solid trabeculated transverse process and facet fusions bilaterally; grade II: thick fusion mass on one side and difficult to visualize on the other side; grade III: suspected lucency or defect in the fusion mass; grade IV: definite resorption of graft with fatigue of instrumentation. The fusion was defined as grade I or grade II.

\section{Statistical Assessment}

Statistical analysis was performed with SPSS version 17.0. Student's $t$ test was used to compare continuous variables (age, duration of symptoms, operative time, intra-operative blood loss, amount of transfusion, X-ray exposure time, duration of hospital stay postoperative and follow-up period). A chi-square test or a Fisher's exact test was used to compare the differences in gender, preoperative diagnoses, levels of fusion, fusion rate at one year after surgery, and complications. Analysis of pain and functional evaluation (VAS and ODI scores) was carried out by means of an unpaired t-test between groups, and analysis of variance was used within each group to compare the pre- and post-treatment. In all analyses, significance was defined as $\mathrm{p}<0.05$.

\section{Results}

No significant differences existed between the two groups in terms of mean age, duration of symptoms, gender, levels of fusion and preoperative diagnosis (Table 1). None of the patients in the MITLIF group needed to be converted to the open surgery. The mean follow-up period was $20.6 \pm$ 4.5 months for group A and 20.0 \pm 3.3 months for group B $(\mathrm{p}>0.05)$. No significant difference existed in operating time between the two groups $(\mathrm{p}>0.05)$. X-ray exposure time was significantly longer for MITLIF compared to open cases, with an average of $45.3 \pm 11.7 \mathrm{~s}$ in group $\mathrm{A}$ and $28.9 \pm 8.2 \mathrm{~s}$ in group B $(p<0.05)$. However, intraoperative blood loss was significantly less in the MITLIF group compared to open procedures. None of the MITLIF patients required blood transfusions, while $11(28.9 \%)$ patients in the open TLIF group required blood transfusions. Duration of hospital stay postoperative was significantly shorter for MITLIF patients (group A) compared to patients with open TLIF (group B). On postoperative day three, MITLIF patients had significantly less pain compared to patients with the open procedure. No statistical difference existed in preoperative and latest VAS value of back pain (VAS-BP) and leg pain (VAS-LP) (Fig. 2), pre-operative and latest ODI between the two groups.

Fusion status was judged on the radiographs at 12-month follow-up. According to the Bridwell's criterion, there were 24 grade I cases, 17 grade II cases, and three grade III cases in group A, and 20 grade I cases, 15 grade II cases, and three grade III cases in group B. The fusion rate (grades I and II) was $93.2 \%$ in group A and $92.1 \%$ in group B with no statistical difference $(p>0.05)$. The patients of grade III are still under follow-up. A representative case is shown in Fig. 3.

Small dural tears occurred in three patients (two patients in group A and one patient in group B). The overlying fascia was closed tightly without additional exposure and repair. Postoperatively, the patients remained strictly supine in bed, and cerebrospinal fluid leakage lasted less than a week without any neurological sequelae or wound complication. Five patients (two patients in group A and three patients in group B) who suffered from superficial wound infection were successfully treated with oral antibiotic therapy and dressing change. Postoperative radiographs showed one pair of screws was overlong and penetrating the front cortical bone of vertebra in one patient in group A (Fig. 4), and no additional symptoms (such as abdominal pain) were observed during the follow-up period in this patient. No device-related complications, such as

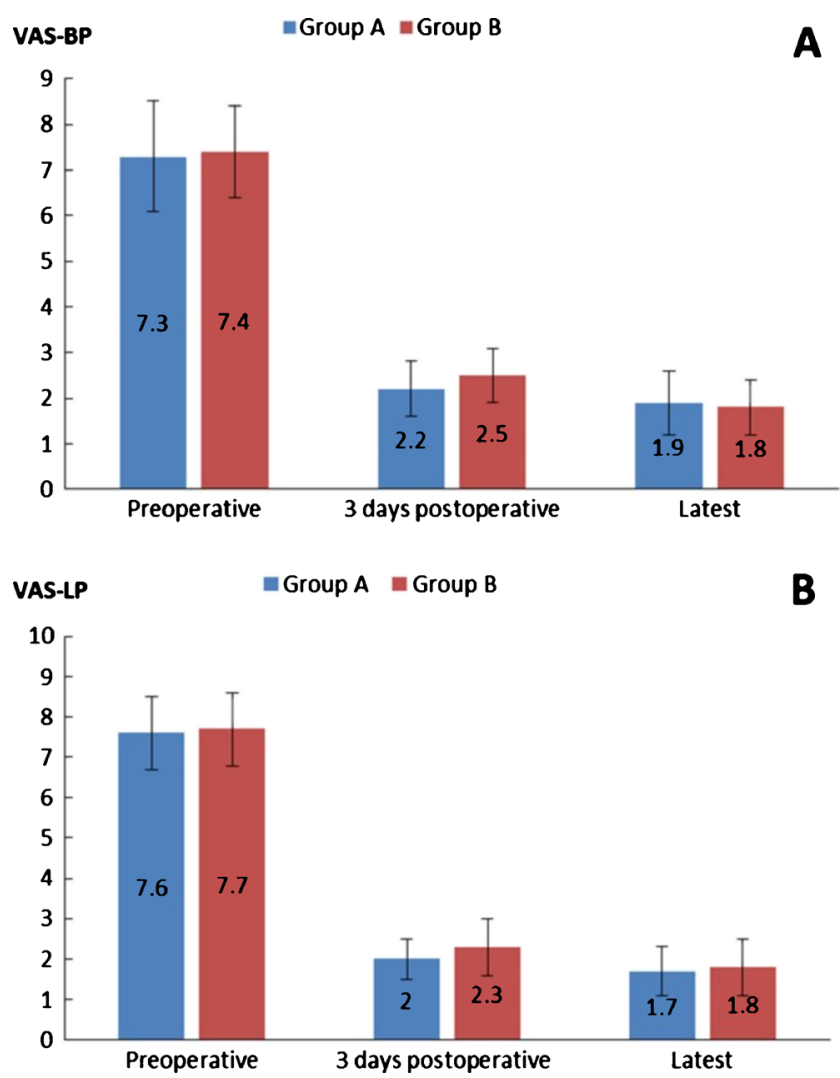

Fig. 2 Change in visual analog scores (VAS) after surgery. a Visual analog score for back pain (VAS-BP) pre-operatively, three days after surgery and at the latest follow-up. b Visual analog score for leg pain (VASLP) preoperatively, three days after surgery and at the latest follow-up 
Fig. 3 A 65-year-old man was admitted to our hospital for low back pain and intermittent claudication for almost 2 years. a, b, c, $\mathbf{d}$ The pre-operative image data of the patient showed lumbar stenosis combined with instability in L4/5 level and degenerative disc disease in L5/S1 level. e, $\mathbf{f}$ AP and lateral radiographs showing the internal fixation and cage the third day after MITLIF. $\mathbf{g}, \mathbf{h}$ CT scan the third day after MITLIF. i, j AP and lateral radiographs showing a solid fusion 12 months after surgery

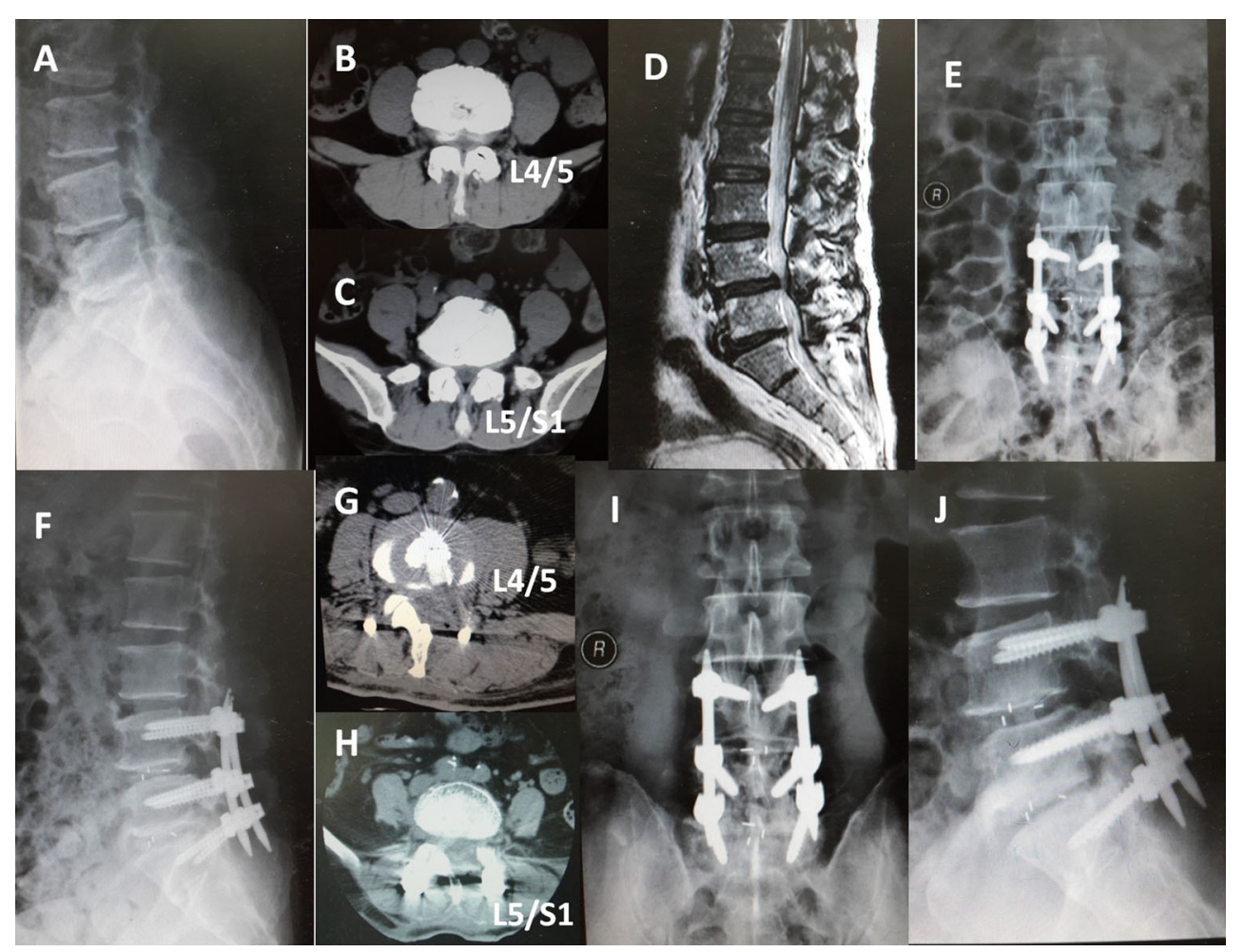

hardware loosening or breakage, cage migration or neurological injury due to violation of the pedicle cortex by the screws occurred in either group. The total complication rate was $11.4 \%$ for group A and $12.1 \%$ for group B. When comparing complications, no significant difference was observed between the groups $(\mathrm{p}>0.05)$. All the details are illustrated in Table 2 .

\section{Discussion}

TLIF, first described by Harms and Rolinger [16], is currently used increasingly as an alternative procedure for lumbar posterolateral fusion or posterior lumbar interbody fusion (PLIF)
[14, 17-19]. As with the PLIF procedure, TLIF offers a number of potential benefits, including increased fusion surface area, complete access for medial and lateral decompression and restoration of intervertebral body height [20]. In addition, TLIF reduces the complications associated with PLIF, because it does not require retraction of the dura or nerve roots, eliminates epidural scarring, and reduces intraoperative bleeding [21-23].

However, in the conventional midline approach, extensive muscle dissection and retraction leads to subsequent denervation and atrophy of the back muscles and may contribute to postoperative pain syndrome, such as fusion disease [24-26]. MITLIF, which is supposed to solve the above-mentioned
Fig. 4 Postoperative X-ray and CT showed one pair of screws was overlong, penetrating the front cortical bone of vertebra in one patient in group $\mathrm{A}$

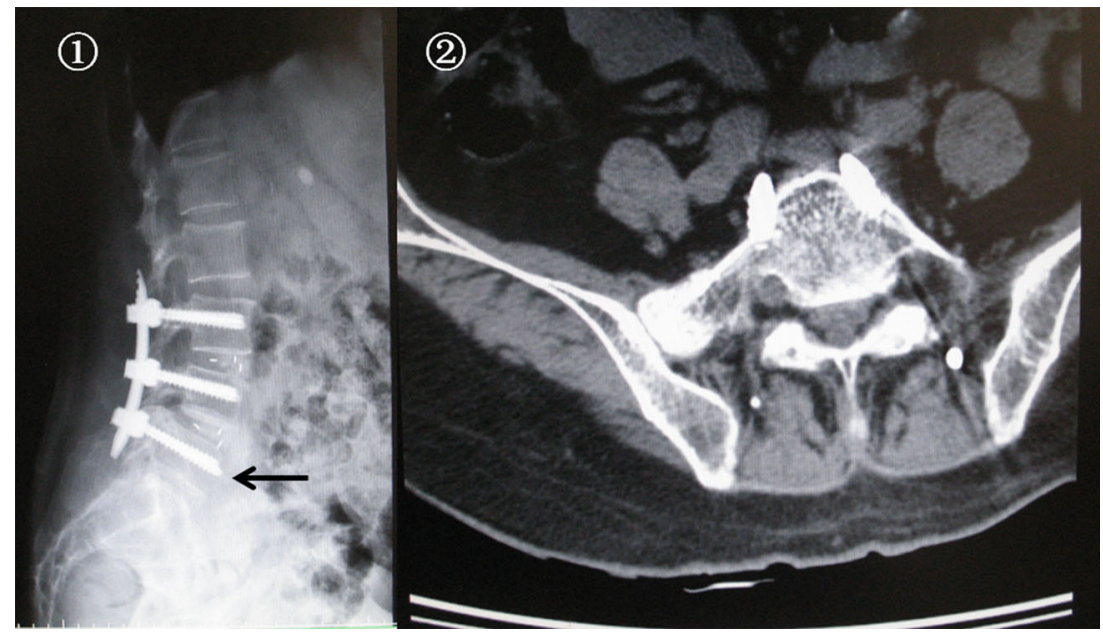


Table 2 Comparison of clinical data between group A and group B

\begin{tabular}{|c|c|c|c|}
\hline Clinical data & Group A & Group B & $\mathrm{P}$ \\
\hline Operating time (min) & $195.5 \pm 28.0$ & $186.6 \pm 23.4$ & 0.13 \\
\hline Intra-operative blood loss (ml) & $248.4 \pm 94.3$ & $576.3 \pm 176.2$ & 0.00 \\
\hline Amount of transfusion (ml) & $0 \pm 0$ & $94.7 \pm 165.9$ & 0.00 \\
\hline X-ray exposure time (s) & $45.3 \pm 11.7$ & $28.9 \pm 8.2$ & 0.00 \\
\hline $\begin{array}{l}\text { Duration of hospital stay } \\
\text { postoperative (d) }\end{array}$ & $9.3 \pm 3.7$ & $12.1 \pm 3.6$ & 0.00 \\
\hline Follow-up period (m) & $20.6 \pm 4.5$ & $20.0 \pm 3.3$ & 0.47 \\
\hline \multicolumn{4}{|l|}{ VAS-BP } \\
\hline Preoperative & $7.3 \pm 1.2$ & $7.4 \pm 1.0$ & 0.61 \\
\hline Three days postoperative & $2.2 \pm 0.6$ & $2.5 \pm 0.6$ & 0.02 \\
\hline Latest & $1.9 \pm 0.7$ & $1.8 \pm 0.6$ & 0.65 \\
\hline \multicolumn{4}{|l|}{ VAS-LP } \\
\hline Preoperative & $7.6 \pm 0.9$ & $7.7 \pm 0.9$ & 0.83 \\
\hline Three days postoperative & $2.0 \pm 0.5$ & $2.3 \pm 0.7$ & 0.03 \\
\hline Latest & $1.7 \pm 0.6$ & $1.8 \pm 0.7$ & 0.53 \\
\hline \multicolumn{4}{|l|}{ ODI (\%) } \\
\hline Preoperative & $43.7 \pm 4.3$ & $44.3 \pm 5.2$ & 0.58 \\
\hline Latest & $16.5 \pm 2.0$ & $15.9 \pm 1.9$ & 0.17 \\
\hline Fusion rate $(\%)$ & $93.2 \%(41 / 44)$ & $92.1 \%(35 / 38)$ & 0.97 \\
\hline \multicolumn{4}{|l|}{ Complications } \\
\hline Dura tear & 2 & 1 & 1.00 \\
\hline Superficial wound infection & 2 & 3 & 0.66 \\
\hline Overlong screws & 1 & 0 & 1.00 \\
\hline Total complications & $5(11.4 \%)$ & $4(12.1 \%)$ & 1.00 \\
\hline
\end{tabular}

VAS-BP visual analog score for back pain, VAS-LP visual analog score for leg pain, ODI Oswestry Disability Index

problems, has become a popular and well-established technique nowadays. Compared with the open procedure, MITLIF appears to achieve similar fusion rates but with the merits of minimizing blood loss and tissue injury, causing smaller wounds, increasing the speed of recovery and reducing postoperative pain $[6-9,27]$. The results of studies concerning single-level MITLIF have been reported, whereas the comparison studies of MITLIF and open TLIF in two-level degenerative lumbar disease have not yet been published.

Early studies on MITLIF reported longer operating time [7, 8, 10]. Foley et al. [28] suggested that MITLIF was more difficult to detect surgical landmarks during operation, because the operating area or space was narrow and limited, and it took surgeons a longer time to learn the surgical techniques and procedures of MITLIF. They also indicated longer operation time required for MITLIF than that for conventional fusion. This is probably due to longer time required for neural decompression and percutaneous screw placement. In this light, there have been various attempts to shorten the operation time of MITLIF, and Min and colleagues [29, 30] reported that it was possible to decrease operation time statistically significantly by a unilateral approach, compared with a bilateral approach. Furthermore, it was thought that the operation time could be shortened as the surgeon gets more familiar with the surgical procedures and techniques of MITLIF. Similarly, a unilateral approach for bilateral decompression was used in our surgery, and we managed to achieve shorter operating time for MITLIF cases. In our study, no significant difference existed in operating time between the two groups. This might be due to the fact that we have overcome the initial stage of the MITLIF learning curve.

Satisfactory clinical outcomes of single level MITLIF have been reported. Potter et al. [14] reported that satisfactory clinical outcomes were shown in $80 \%$ of 100 patients who underwent a single MITLIF and were followed-up over two years. Foley et al. [28] reported improvements in ODI score from 55 points pre-operatively to 11 points postoperatively. In this study, clinical outcomes, determined by VASBP, VAS-LP and ODI scores, were significantly improved postoperatively compared to pre-operatively, and were not significantly different between the two groups, except VASBP and VAS-LP scores three days after surgery. We attribute these differences to less tissue trauma and structure damage during surgery in MITLIF, while still safely accomplishing the goals of the conventional open TLIF. Intra-operative blood loss and duration of postoperative hospital stay of the MITLIF group in our study were obviously superior to open TLIF. No patients in the MITLIF group needed blood transfusions perioperatively, while in the conventional open TLIF group, 11 patients received blood transfusions during surgery. Thus, MITLIF obviously reduced the need for blood transfusions and its associated risks.

Based on the Bridwell's posterior fusion grading system, there were no significant differences in the fusion rates between MITLIF and open TLIF patients in the study. A total of $93.2 \%(41 / 44)$ of the MITLIF group and $92.1 \%(35 / 38)$ of the open TLIF achieved grade I or grade II fusion $(\mathrm{p}>0.05)$. The complications included dura tear, superficial wound infection and overlong screws. The overall complication rate for MITLIF (11.4\%) is nearly the same for the open group $(12.1 \%)(\mathrm{p}>0.05)$. No device-related complications, such as hardware loosening or breakage, cage migration, or neurological injury due to violation of the pedicle cortex by the screws, occurred in either group. No radiological signs of adjacent segment degeneration (ASD) were found in both groups.

Minimally invasive spinal fusion using the MITLIF technique is efficacious and safe. However, the need for intraoperative fluoroscopy poses disadvantages. Occupational radiation exposure is not a negligible issue in daily clinics, and consensus that its hazards must be acknowledged and better understood has been made by the major American societies of physicians [31]. It has been indicated that radiation had a certain randomness or linear relationship with the occurrence of cancer and cataracts [32], and recently the International Commission on Radiological Protection recommended that 
for occupational exposure in planned exposure situations, the equivalent dose limit for the lens of the eye be reduced from $150 \mathrm{mSv}$ to $20 \mathrm{mSv}$ in a year based on robust epidemiological evidence [33]. Therefore, we must take complete protection such as wearing leaded apron and glasses during surgery and mitigate radiation exposure to the greatest extent possible. Our study indeed found much longer X-ray exposure time in the MITLIF group. At this point, the surgeons' experience in minimally invasive surgery should not be neglected. Compared to open TLIF, MITLIF is technically more challenging, because the surgery involves a much smaller operative field and lacks of visualization of the bony landmarks, and the tactile feedback can also hinder the accurate decompression, interbody fusion and percutaneous pedicle screw placement. This technique, as with all minimally invasive surgical strategies, requires a steep learning curve. This is associated with significantly longer $\mathrm{X}$-ray exposure time.

The present study is not without limitations. First, the sample size was small and the follow-up periods were short. Finally, postoperative MRI or CT scans were not performed in all patients, so deviations would exist when assessing the fusion status and ASD.

\section{Conclusion}

MITLIF was superior to the traditional open TLIF in the management of two-level degenerative lumbar diseases. The minimally invasive procedure was superior in terms of postoperative back pain and leg pain, intra-operative blood loss, transfusion and duration of hospital stay postoperatively. However, it required much more radiation exposure.

Acknowledgments We thank Xia Zhang, a nurse in our hospital, for her excellent daily work in our surgery.

Disclosure The authors declare that they have no competing interests. No funds were received in support of this work. No benefits in any form have been or will be received from a commercial party related directly or indirectly to the subject of this manuscript.

\section{References}

1. Sethi A, Lee S, Vaidya R (2009) Transforaminal lumbar interbody fusion using unilateral pedicle screws and a translaminar screw. Eur Spine J 18:430-434

2. Lee KH, Yue WM, Yeo W, Soeharno H, Tan SB (2012) Clinical and radiological outcomes of open versus minimally invasive transforaminal lumbar interbody fusion. Eur Spine J 21:2265-2270

3. Lowe TG, Tahernia AD, O'Brien MF, Smith DA (2002) Unilateral transforaminal posterior lumbar interbody fusion (TLIF): indications, technique, and 2-year results. J Spinal Disord Tech 15:31-38

4. Rosenberg WS, Mummaneni PV (2001) Transforaminal lumbar interbody fusion: technique, complications, and early results. Neurosurgery 48:569-574, discussion 574-575
5. Foley KT, Lefkowitz MA (2002) Advances in minimally invasive spine surgery. Clin Neurosurg 49:499-517

6. Schwender JD, Holly LT, Rouben DP, Foley KT (2005) Minimally invasive transforaminal lumbar interbody fusion (TLIF): technical feasibility and initial results. J Spinal Disord Tech 18:S1-6

7. Peng CW, Yue WM, Poh SY, Yeo W, Tan SB (2009) Clinical and radiological outcomes of minimally invasive versus open transforaminal lumbar interbody fusion. Spine 34:1385-1389

8. Villavicencio AT, Burneikiene S, Roeca CM, Nelson EL, Mason A (2010) Minimally invasive versus open transforaminal lumbar interbody fusion. Surg Neurol Int 1:12

9. Wang J, Zhou Y, Zhang ZF, Li CQ, Zheng WJ, Liu J (2010) Comparison of one-level minimally invasive and open transforaminal lumbar interbody fusion in degenerative and isthmic spondylolisthesis grades 1 and 2. Eur Spine J 19:1780-1784

10. Shunwu F, Xing Z, Fengdong Z, Xiangqian F (2010) Minimally invasive transforaminal lumbar interbody fusion for the treatment of degenerative lumbar diseases. Spine 35:1615-1620

11. Kai Z, Wei S, Chang-Qing Z, Hua L, Wei D, You-Zhuan X, XiaoJiang S, Jie Z (2013) Unilateral versus bilateral instrumented transforaminal lumbar interbody fusion in two-level degenerative lumbar disorders: a prospective randomised study. Int Orthop 2013 Aug 6. [Epub ahead of print]

12. Gu G, Zhang H, He S, Jia J, Fu Q, Zhou X (2013) Preoperative localization methods for minimally invasive surgery in lumbar spine: comparisons between a novel method and conventional methods. J Spinal Disord Tech 26:E277-277E280

13. Yucesoy K, Yuksel KZ, Baek S, Sonntag VK, Crawford NR (2008) Biomechanics of unilateral compared with bilateral lumbar pedicle screw fixation for stabilization of unilateral vertebral disease. $\mathrm{J}$ Neurosurg Spine 8:44-51

14. Potter BK, Freedman BA, Verwiebe EG, Hall JM, Polly DW Jr, Kuklo TR (2005) Transforaminal lumbar interbody fusion: clinical and radiographic results and complications in 100 consecutive patients. J Spinal Disord Tech 18:337-346

15. Bridwell KH, Lenke LG, McEnery KW, Baldus C, Blanke K (1995) Anterior fresh frozen structural allografts in the thoracic and lumbar spine. Do they work if combined with posterior fusion and instrumentation in adult patients with kyphosis or anterior column defects. Spine 20:1410-1418

16. Harms J, Rolinger H (1982) A one-stager procedure in operative treatment of spondylolistheses: dorsal traction-reposition and anterior fusion (author's transl). Z Orthop Ihre Grenzgeb 120:343-347

17. Blume HG (1985) Unilateral posterior lumbar interbody fusion: simplified dowel technique. Clin Orthop Relat Res 193:75-84

18. Harms JG, Jeszenszky D (1998) In Process Citation. Oper Orthop Traumatol 10:90-102

19. Taneichi H, Suda K, Kajino T, Matsumura A, Moridaira H, Kaneda K (2006) Unilateral transforaminal lumbar interbody fusion and bilateral anterior-column fixation with two Brantigan I/F cages per level: clinical outcomes during a minimum 2 -year follow-up period. J Neurosurg Spine 4:198-205

20. Branch CL Jr (1996) The case for posterior lumbar interbody fusion. Clin Neurosurg 43:252-267

21. DiPaola CP, Molinari RW (2008) Posterior lumbar interbody fusion. J Am Acad Orthop Surg 16:130-139

22. German JW, Foley KT (2005) Minimal access surgical techniques in the management of the painful lumbar motion segment. Spine 30: S52-59

23. Xiao Y, Li F, Chen Q (2010) Transforaminal lumbar interbody fusion with one cage and excised local bone. Arch Orthop Trauma Surg 130: 591-597

24. Gejo R, Matsui H, Kawaguchi Y, Ishihara H, Tsuji H (1999) Serial changes in trunk muscle performance after posterior lumbar surgery. Spine 24:1023-1028 
25. Sihvonen T, Herno A, Paljarvi L, Airaksinen O, Partanen J, Tapaninaho A (1993) Local denervation atrophy of paraspinal muscles in postoperative failed back syndrome. Spine 18: 575-581

26. Weber BR, Grob D, Dvorak J, Muntener M (1997) Posterior surgical approach to the lumbar spine and its effect on the multifidus muscle. Spine 22:1765-1772

27. Schizas C, Tzinieris N, Tsiridis E, Kosmopoulos V (2009) Minimally invasive versus open transforaminal lumbar interbody fusion: evaluating initial experience. Int Orthop 33:1683-1688

28. Foley KT, Holly LT, Schwender JD (2003) Minimally invasive lumbar fusion. Spine 28:S26-35

29. Min S, Hwang S (2009) Minimal invasive unilateral transforaminal lumbar interbody fusion by sublaminar decompression - comparison to bilateral approach. J Korean Orthop Assoc 44:7682
30. Min S, Lee D (2009) Minimally invasive transforaminal lumbar interbody fusion in patients with low grade spondylolisthesis - comparison of the unilateral and bilateral approaches. J Korean Orthop Assoc 44:429435

31. Klein LW, Miller DL, Balter S, Laskey W, Haines D, Norbash A, Mauro MA, Goldstein JA (2009) Occupational health hazards in the interventional laboratory: time for a safer environment. Radiology 250:538-544

32. Brenner DJ, Doll R, Goodhead DT, Hall EJ, Land CE, Little JB, Lubin JH, Preston DL, Preston RJ, Puskin JS, Ron E, Sachs RK, Samet JM, Setlow RB, Zaider M (2003) Cancer risks attributable to low doses of ionizing radiation: assessing what we really know. Proc Natl Acad Sci USA 100:13761-13766

33. Thorne MC (2012) Regulating exposure of the lens of the eye to ionising radiations. J Radiol Prot 32:147-154 